\title{
Similarities and differences between selective and nonselective BAFF blockade in murine SLE
}

\author{
Meera Ramanujam, ${ }^{1}$ Xiaobo Wang, ${ }^{2}$ Weiqing Huang, ${ }^{1}$ Zheng Liu, ${ }^{1}$ Lena Schiffer, ${ }^{1}$ Haiou Tao, ${ }^{1}$ \\ Daniel Frank, ${ }^{1}$ Jeffrey Rice, ${ }^{1}$ Betty Diamond, ${ }^{1}$ Karl O.A. Yu, ${ }^{2}$ Steven Porcelli, ${ }^{2}$ and Anne Davidson ${ }^{1}$ \\ ${ }^{1}$ Departments of Medicine and Microbiology, Columbia University, New York, New York, USA. ${ }^{2}$ Department of Microbiology and Immunology, \\ Albert Einstein College of Medicine, New York, New York, USA.
}

\begin{abstract}
B cells have multiple roles in immune activation and inflammation separate from their capacity to produce antibodies. $B$ cell depletion is currently under intense investigation as a therapeutic strategy for autoimmune diseases. The TNF family members B cell-activating factor of the TNF family (BAFF) and its homolog A proliferation-inducing ligand (APRIL) are $B$ cell survival and differentiation factors and are therefore rational therapeutic targets. We compared the effects of BAFF receptor-Ig, which blocks only BAFF, with those of transmembrane activator and calcium modulator ligand interactor-Ig, which blocks both BAFF and APRIL, in a murine SLE model. Both reagents prolonged the life of NZB/W F1 mice when given either before or after disease onset. Many immunologic effects of the 2 reagents were similar, including B cell and B cell subset depletion and prevention of the progressive $T$ cell activation and dendritic cell accumulation that occurs with age in NZB/W mice without substantial effects on the emergence of the IgG anti-double-stranded DNA response. Furthermore, both reagents inhibited the $T$ cell-independent marginal zone $B$ cell response to particulate antigen delivered i.v., but not the B1 B cell response to the same antigen delivered i.p. In contrast, blockade of both BAFF and APRIL, but not blockade of BAFF alone, reduced the serum levels of IgM antibodies, decreased the frequency of plasma cells in the spleen, and inhibited the IgM response to a $T$ cell-dependent antigen. The differences between selective and nonselective BAFF blockade are relevant to the choice of a BAFF blocking agent for the treatment of autoimmune and malignant diseases.
\end{abstract}

\section{Introduction}

It is increasingly recognized that B cells have multiple functions that contribute to the pathogenesis of autoimmunity. They produce autoantibodies that mediate tissue injury, they function as antigenpresenting cells that present epitopes of self antigen to autoreactive $\mathrm{T}$ cells, and they produce soluble mediators involved in the organization of lymphoid tissues and in the initiation and perpetuation of inflammatory processes (1). In some autoimmune diseases, B cells migrate to inflamed sites, where they act as local effector cells $(2,3)$.

The TNF-like molecule B cell-activating factor of the TNF family (BAFF; TNFSF13b) is a key B cell survival factor, and its 3 receptors (transmembrane activator and calcium modulator ligand interactor [TACI; TNFRSF13b], B cell maturation antigen [BCMA; TNFRSF17], and BAFF receptor [BAFF-R; TNFRSF13c]) are variably expressed on $B$ cells during their differentiation (4). A proliferation-inducing ligand (APRIL; TNFSF13a), a molecule homologous to BAFF, binds only to TACI and BCMA and shares many functions in common with BAFF, although it cannot facilitate survival of transitional B cells, a function that depends on the interaction of BAFF with BAFF-R (5). Serum levels of BAFF and APRIL are increased in autoimmune diseases, including SLE and rheumatoid arthritis $(6,7)$, and blockade of BAFF and APRIL

Nonstandard abbreviations used: Ad-, adenovirus; APRIL, A proliferation-inducing ligand; BAFF, B cell-activating factor of the TNF family; BAFF-R, BAFF receptor; BCMA, B cell maturation antigen; dsDNA, double-stranded DNA; KLH, keyhole limpet hemocyanin; NP, 4-hydroxy-3-nitrophenylacetyl; PC, phosphorylcholine; T1, transitional type 1; TACI, transmembrane activator and calcium modulator ligand interactor. Conflict of interest: The authors have declared that no conflict of interest exists. Citation for this article: J. Clin. Invest. doi:10.1172/JCI26385. using soluble fusion proteins of BAFF receptors prevents autoimmunity in animal models of disease (8-11).

A number of different BAFF antagonists are in early clinical trials for human autoimmune diseases. Some, such as BAFF-R-Ig and anti-BAFF, selectively block only BAFF, whereas others, such as TACI-Ig, block both BAFF and APRIL (12). Since plasma cells predominantly express BCMA and TACI that bind to both BAFF and APRIL $(13,14)$, these differences may be physiologically important. Furthermore, the mechanism of action of these therapeutic reagents needs to be explored in the setting of autoimmunity because intrinsic B cell hyperreactivity, the provision of excess $T$ cell help, and the presence of inflammatory mediators may alter the normal dependence of B cells on BAFF or APRIL and thus the response to blockade.

Our goal in this study was to examine the immunologic effects of selective and nonselective BAFF blockade in a murine model of SLE. Our results show that although both BAFF-R-Ig and TACI-Ig prevented the onset of SLE in this model, there were significant differences in the effects of the 2 reagents on the survival of plasma cells in the spleen and bone marrow. These differences may affect the type of disease that will be responsive to these reagents as well as their immunosuppressive potential.

\section{Results}

Expression of BAFF-R-Ig and TACI-Ig fusion proteins. Fully murine BAFF-R-Ig and TACI-Ig were expressed using recombinant adenoviruses. BAFF-R-Ig is a monomer on SDS-PAGE, whereas TACI-Ig is a covalently linked dimer (Figure 1A). (15). There is little difference between TACI-Ig and BAFF-R-Ig with respect to half-life (data 

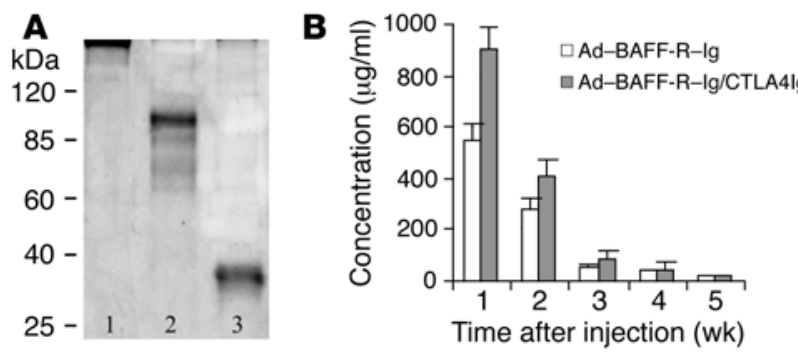

not shown), relative affinity for BAFF, or the ability to inhibit the binding of BAFF to membrane-bound BAFF-R (Supplemental Figure 1; available online with this article; doi:10.1172/JCI26385DS1). As expected, BAFF-R-Ig bound only to BAFF, whereas TACI-Ig bound to both BAFF and APRIL in ELISA assays (data not shown). Administration of a single dose of adenovirus expressing BAFF-R-Ig to 18- to 20-week-old NZB/W F1 mice resulted in expression of BAFF-R-Ig in the serum for approximately 6 weeks. In order to determine whether the addition of an agent that blocks $T$ cell activation would be synergistic with BAFF-R-Ig, the adenovirus was also administered together with the costimulatory antagonist CTLA4Ig. The addition of 2 weeks of treatment with CTLA4Ig to BAFF-R-Ig adenovirus (Ad-BAFF-R-Ig/CTLA4Ig) resulted in an increased initial level of expression of BAFF-R-Ig in the serum, but did not significantly prolong the duration of expression (Figure $1 \mathrm{~B}$ ).

Clinical effects of BAFF-R-Ig in NZB/W F1 mice. The clinical effects of TACI-Ig in NZB/W F1 mice have been previously described in detail (10). Treatment of 18- to 20-week-old NZB/W F1 mice with Ad-BAFF-R-Ig with or without CTLA4Ig resulted in a highly significant delay of 6 months in proteinuria onset and death compared with controls (Figure 2, A and B). We have previously reported that administration of Ad-TACI-Ig to NZB/W F1 mice causes a decrease in serum IgM levels that is sustained for up to 6 months after treatment (10). In contrast, treatment with Ad-BAFF-R-Ig did not alter the serum levels of either IgM (Figure 3A) or IgM antiDNA antibodies (data not shown). Ad-BAFF-R-Ig alone did not alter the serum levels of IgG (Figure 3B) or of IgG isotypes; however, a transient decrease of serum IgG1 levels was noted with combination Ad-BAFF-R-Ig/CTLA4Ig (data not shown). The appearance of IgG anti-DNA antibodies in the serum of Ad-BAFF-R-Ig-treated mice was delayed by 6 weeks, which was equivalent to the time that the fusion protein was expressed in the serum (Figure 2C). Furthermore, IgG deposits and complement could be detected in the kidneys of Ad-BAFF-R-Ig-treated mice at the age of 36 weeks, well before the onset of clinical disease, indicating that the autoantibod-

\section{Figure 2}

Ad-BAFF-R-Ig prolongs survival of NZB/W F1 mice. Kaplan-Meier plots of proteinuria-free survival $(\mathbf{A})$ and overall survival $(\mathbf{B})$ in treated NZB/W F1 mice aged 18-20 weeks. Mice treated with Ad-BAFF-R-Ig alone or together with CTLA4lg had prolonged survival $(P<0.0001$, Ad-BAFF-R-Ig versus control; $P<0.003$, Ad-BAFF-R-Ig/CTLA4Ig versus $A d-L a c Z / C T L A 4 I g)$ and prolonged proteinuria-free survival compared with untreated or irrelevant adenovirus controls $(P<0.001$, Ad-BAFF-R-Ig versus untreated; $P<0.01$, Ad-BAFF-R-Ig/CTLA4Ig versus Ad-LacZ/CTLA4Ig). (C) Time of appearance of high-titer IgG anti-dsDNA antibodies. In both Ad-BAFF-R-Ig- and Ad-BAFF-R-Ig/ CTLA4Ig-treated groups $(P<0.0013$ and $P<0.0139$, respectively), there was a delay of approximately 6 weeks in the appearance of $\lg G$ anti-dsDNA antibodies compared with the relevant control.

\section{Figure 1}

BAFF-R-Ig serum levels in SCID mice. (A) SDS-PAGE of immunoprecipitated control mouse IgG2a (lane 1) and sera from SCID mice injected with either Ad-TACI-Ig (lane 2) or Ad-BAFF-R-Ig (lane 3). $\mathrm{TACl}-\mathrm{lg}$ is a $94-\mathrm{kDa}$ dimer; BAFF-R-Ig is a $37-\mathrm{kDa}$ monomer. (B) Mean \pm SD serum BAFF-R-Ig levels over time in NZB/W F1 mice ( $n=10$ per group) injected at day 0 with $10^{9}$ pfu Ad-BAFF-R-Ig alone or together with 6 doses of $100 \mu \mathrm{g}$ CTLA4Ig.

ies had pathogenic potential (Figure 4). This is similar to what we have previously reported with Ad-TACI-Ig (10).

Analysis of B and T cell phenotypes. Spleens from Ad-BAFF-R-Igtreated mice were examined at 36 weeks of age. The spleens were significantly smaller and contained fewer cells than the spleens of control 16- and 36-week-old NZB/W F1 mice. The B cells had a phenotype similar to that of mice treated with Ad-TACI-Ig (10), namely an intact transitional type 1 (T1) population, a decrease in the number and proportion of marginal zone and follicular B cells, and a decrease in the number of activated and class-switched $\mathrm{B}$ cells (Tables 1 and 2 ). $\mathrm{CD}^{+} \mathrm{B}$ cells were examined in the mice treated with combination Ad-BAFF-R-Ig/CTLA4Ig and were not depleted. Similarly, peritoneal $\mathrm{CD}^{+} \mathrm{B}$ cells were not depleted 8 weeks after treatment with either Ad-TACI-Ig or Ad-BAFF-R-Ig (data not shown).

Mice treated with Ad-BAFF-R-Ig and analyzed at 36 weeks of age had a marked decrease in the number of activated and memory CD4 $T$ cells compared with age-matched controls, indicating a significant inhibitory effect of Ad-BAFF-R-Ig treatment on CD4 T cell activa-
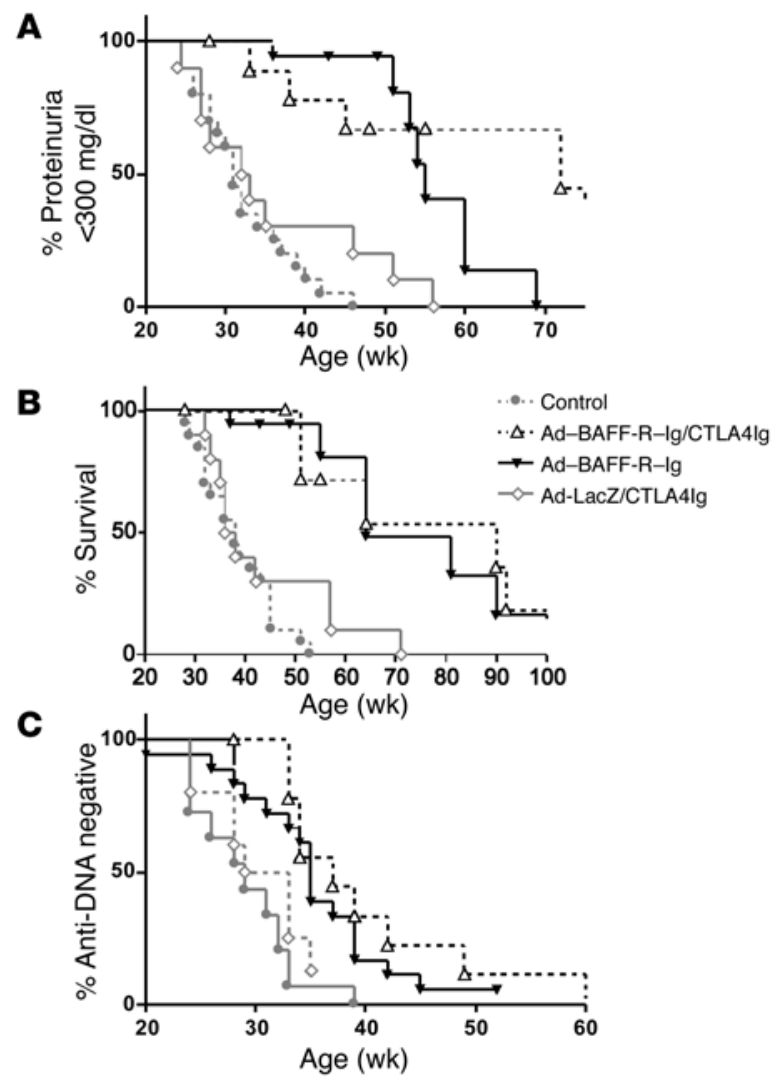

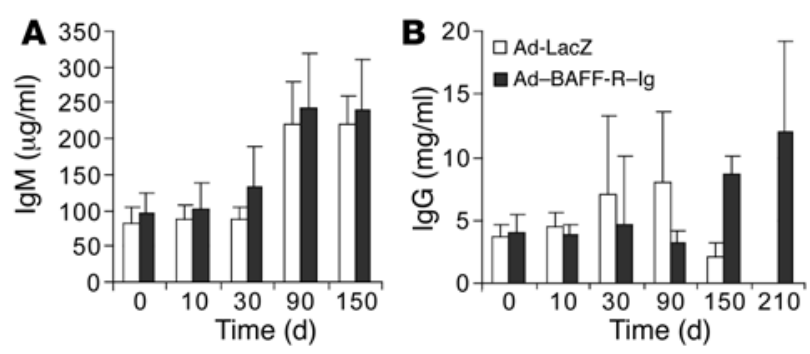

C

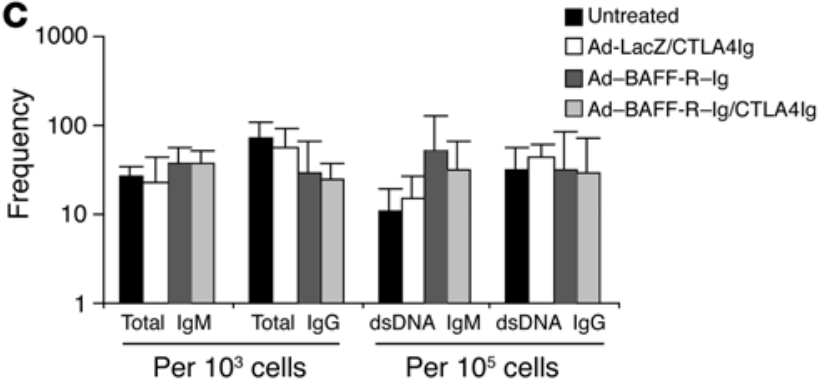

D

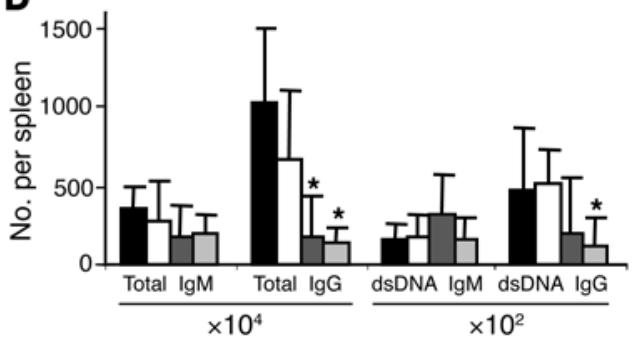

tion and proliferation. Similarly, Ad-BAFF-R-Ig-treated mice did not accumulate increased numbers of CD11c/CD11b dendritic cells over time as seen in age-matched controls (Tables 1 and 2).

Analysis of B cell Ig secretion. To enumerate the frequency of Ig-secreting cells and autoantibody-secreting cells, ELISpot assay was performed on equal numbers of spleen cells from treated and control mice. At 36 weeks of age, the frequency of IgG-secreting cells in the spleen was not significantly different from controls (Figure 3C), but due to the small spleen size, the total number of IgG-secreting cells per spleen was significantly lower in Ad-BAFF$\mathrm{R}-\mathrm{Ig}$-treated mice than in controls (Figure 3D). No preferential depletion of IgG anti-DNA secreting B cells was observed (Figure $3 \mathrm{C})$. The frequency and total number of IgM-secreting B cells was not altered by Ad-BAFF-R-Ig (Figure 3, C and D).

Induction of remission by $A d-B A F F-R-I g$ and CTLA4Ig. We have previously reported that the combination of Ad-TACI-Ig and CTLA4Ig

\section{Figure 3}

Ad-BAFF-R-Ig does not alter serum Ig levels. Mean \pm SD serum IgM (A) and IgG (B) in Ad-BAFF-R-Ig-treated NZB/W F1 mice were not significantly different from control Ad-LacZ-treated mice. Low IgG levels at 150 days in control mice reflect losses due to proteinuria in these mice. (C) The frequency of IgG-secreting cells per $10^{3}$ or $10^{5}$ total spleen cells (4-5 per group) was determined by ELISpot assay and was not significantly different in treated mice compared with controls. (D) Mean + SD number of IgM or IgG antibody-secreting B cells per spleen from control and treated NZB/W F1 mice. There was a significant reduction in the number of IgG-secreting cells in both Ad-BAFFR-lg-treated groups $(P<0.03)$ and of IgG anti-dsDNA-secreting cells in the Ad-BAFF-R-Ig/CTLA4Ig group $(P<0.04)$ compared with the relevant controls. Asterisks indicate significant differences.

induces remission of established disease in NZB/W F1 mice (10). The combination of Ad-BAFF-R-Ig and CTLA4Ig was similarly able to prolong life span in 28- to 30-week-old NZB/W F1 mice (Figure 5, A and B). Compared with Ad-LacZ/CTLA4Ig-treated mice, Ad-BAFF$\mathrm{R}-\mathrm{Ig} / \mathrm{CTLA} 4 \mathrm{Ig}$-treated mice had significant depletion of total spleen cells $\left(8.85 \pm 7.08 \times 10^{7}\right.$ versus $\left.20.42 \pm 12.74 \times 10^{7} ; P<0.03\right)$, total B cells $\left(3.88 \pm 3.36 \times 10^{7}\right.$ versus $\left.10.98 \pm 3.85 \times 10^{7} ; P<0.02\right)$, and activated B cells $\left(0.71 \pm 0.82 \times 10^{7}\right.$ versus $\left.1.67 \pm 1.08 \times 10^{7} ; P<0.05\right)$ at sacrifice without significant changes in the number or activation state of CD4 T cells. As observed in younger mice, there was no effect of Ad-BAFF-R-Ig/CTLA4Ig on the serum levels of IgM or IgM antiDNA antibodies. The effect on IgG anti-DNA antibodies was variable, with temporary decreases of 4-5 weeks occurring in half the mice (data not shown). Histologic analysis of the kidneys showed a decrease in glomerulonephritis and tubular damage in both groups of treated mice (Figure 5C).

The effect of BAFF-R-Ig and TACI-Igon Tcell-dependent and-independent $I g M$ responses. Serum IgM in normal mice derives from several B cell subsets including B1 cells, marginal zone B cells, and activated extrafollicular B2 cells. To determine whether BAFF-R-Ig and TACI-Ig had different effects on the activation of these $B$ cell subsets in a protective immune response, we immunized treated NZB/W F1 mice with $T$ cell-dependent and -independent antigens.

Ad-BAFF-R-Ig-treated mice immunized with the T cell-dependent antigen oxazolone mounted an IgM anti-oxazolone response that was equivalent to that of untreated controls. In contrast, Ad-TACI-Ig-treated mice had a markedly attenuated serum IgM anti-oxazolone response (Figure 6). The IgG1 and IgG2a antioxazolone response was decreased by approximately $50 \%$ by both reagents (Figure 6). Thus, only TACI-Ig inhibited the IgM response to a $T$ cell-dependent antigen, whereas the 2 reagents had similar effects on the class-switched response.

\section{Figure 4}

Ad-BAFF-R-Ig prevents renal inflammation. Histologic analysis of kidneys from 36-week-old control (A, C, and E) and treated (B, D, and F) NZB/W mice. Control kidneys had perivascular interstitial infiltrates (arrows), glomerulonephritis, and tubular casts (A) as well as Ig deposits (E). Ad-BAFF-R-Ig treatment at 18-20 weeks prevented glomerulonephritis and interstitial inflammation ( $B$ and $\mathbf{D}$ ) but did not prevent the deposition of IgG in the glomeruli (F). Magnification, $\times 40(\mathbf{A}-\mathbf{D}) ; \times 10(\mathbf{E}$ and F).
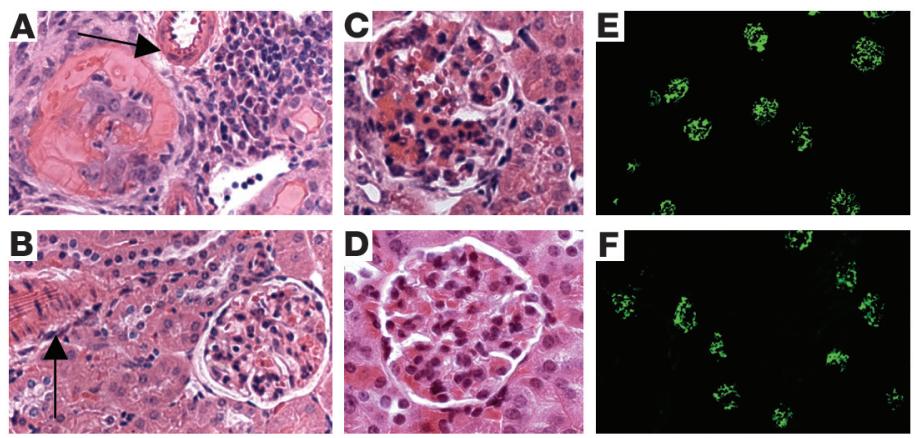
Table 1

Frequency of spleen cell subsets

\begin{tabular}{|c|c|c|c|c|}
\hline Subset & $\begin{array}{c}\text { 16-week-old } \\
\text { NZB/WA } \\
(n=5)\end{array}$ & $\begin{array}{c}\text { 36-week-old } \\
\text { NZB/W } \\
(n=7)\end{array}$ & $\begin{array}{c}\text { 36-week-old } \\
\text { Ad-BAFF-R-Ig/ } \\
\text { CTLA4Ig }(n=5)\end{array}$ & $\begin{array}{l}\text { 36-week-old } \\
\text { Ad-BAFF-R-Ig } \\
(n=4)\end{array}$ \\
\hline CD19 & $38.5 \pm 2.1$ & $49.9 \pm 7.4$ & $23.6 \pm 7.1^{\mathrm{B}}$ & $26.4 \pm 8.9^{B}$ \\
\hline CD19/CD69 & $3.7 \pm 0.4$ & $12.0 \pm 3.1$ & $5.1 \pm 0.4^{\mathrm{B}}$ & $11.1 \pm 0.5$ \\
\hline Follicular & $65.1 \pm 1.3$ & $57.2 \pm 13.7$ & $40.1 \pm 19.5$ & $30.8 \pm 13.2^{c}$ \\
\hline $\mathrm{T} 1$ & $8.2 \pm 1.8$ & $6.6 \pm 1.3$ & $18.1 \pm 9.8$ & $15.6 \pm 4.2$ \\
\hline Marginal zone & $11.8 \pm 2.5$ & $5.7 \pm 2.0$ & $2.8 \pm 0.8^{C}$ & $4.3 \pm 2.8$ \\
\hline $\mathrm{CD}^{+}(\mathrm{B} 1 \mathrm{a})$ & ND & $8.8 \pm 6.5$ & $21.5 \pm 5.9$ & $19.1 \pm 2.1$ \\
\hline IgM-/lgD- (switched) & $5.5 \pm 1.0$ & $16.9 \pm 9.9$ & $16.6 \pm 3.9$ & $29.9 \pm 16.6$ \\
\hline CD4 & $25.3 \pm 1.0$ & $25.5 \pm 3.3$ & $38.8 \pm 5.6^{B}$ & $33.5 \pm 9.7$ \\
\hline CD4/CD69 & $7.4 \pm 2.3$ & $38.0 \pm 9.1$ & $9.6 \pm 2.4^{\mathrm{D}}$ & $13.7 \pm 1.6^{\mathrm{D}}$ \\
\hline CD4/CD44+/CD62L- (memory) & $28.9 \pm 10.1$ & $72.1 \pm 8.9$ & $32.0 \pm 6.3^{B}$ & $42.2 \pm 20.4^{D}$ \\
\hline CD4/CD44-/CD62L+ (naive) & $42.6 \pm 13.5$ & $13.5 \pm 8.6$ & $54.4 \pm 7.6^{B}$ & $40.8 \pm 17.1^{D}$ \\
\hline CD8 & $8.8 \pm 1.6$ & $4.8 \pm 1.5$ & $20.4 \pm 5.1^{\mathrm{B}}$ & $25.4 \pm 2.5^{B}$ \\
\hline CD11c/CD11b & $1.9 \pm 0.3$ & $2.1 \pm 1.1$ & $1.0 \pm 0.4$ & $1.3 \pm 0.5$ \\
\hline
\end{tabular}

Values are mean percent \pm SD. APreviously published in ref. $10 .{ }^{B} P<0.01,{ }^{C} P<0.05, D P<0.02$ versus agematched controls. ND, not determined. and BAFF-R-Ig proteins to more closely approximate therapeutic dosing, marginal zone B cells were depleted by approximately $50 \%$ by both treatments (data not shown). TACI-Ig protein consistently suppressed the serum anti$\mathrm{PC}$ response to a greater degree than did BAFF-R-Ig, and this was associated with lower numbers of idiotype-positive B cells and plasmablasts in the spleen (Figure 9).

Different effects of TACI-Ig and $B A F F-R-I g$ in NZM2410 mice. NZM2410 mice differ from NZB/ W F1 mice in that large numbers of IgG-producing plasma cells reside in the spleen and only few migrate from the germinal centers to the bone marrow (18). In 14-week-old NZM2410 mice, treatment with Ad-TACI-Ig, but not

The IgM response to heat-killed streptococci in normal mice derives from both B1 and marginal zone B cells in the spleen when bacteria are administered i.v., but only from peritoneal B1 $B$ cells when administered i.p. (16). We therefore measured the IgM response to phosphorylcholine (PC) 3 days after i.v. or i.p. administration of heat-killed streptococci. The serum IgM antiPC response of Ad-BAFF-R-Ig- and Ad-TACI-Ig-treated mice following i.p. administration of bacteria was similar to that of control Ad-LacZ-treated mice. ELISpot analysis of peritoneal B cells also showed no difference between treated mice and controls, indicating that B1 cells in the peritoneum of NZB/W F1 mice may function independently of both BAFF and APRIL (Figure 7, A and B). In contrast, both Ad-BAFF-R-Ig and Ad-TACI-Ig suppressed the spleen-derived IgM anti-PC response following i.v. immunization with bacteria (Figure 7, C and D).

To further quantitate the effects of TACI-Ig and BAFF-R-Ig just on marginal zone B cells, these experiments were repeated in C57BL/ 6 mice transgenic for the $\mu$ heavy chain of the anti-PC antibody M167 (17). Transgenic B cells that coexpress the M167 light chain localize to the marginal zones, and the number of $\mathrm{B}$ cells that respond to immunization can be quantitated by flow cytometry using an antiidiotypic antibody (16). Pretreatment of M167 mice with either Ad-TACI-Ig or Ad-BAFF-R-Ig resulted in the loss of $80 \%$ of marginal zone B cells and complete abrogation of the anti-PC response following i.v. immunization with bacteria (Figure $8)$. When the mice were dosed with equal amounts of TACI-Ig these levels remained low for 4 weeks (Figure 10, A and B). ELISpot analysis on spleens and bone marrow at 18 weeks showed that both IgM- and IgG-secreting cells were markedly decreased in the spleens confirmed by immunohistochemistry (Figure 11). Bone marrow IgG-producing plasma cells were also decreased in the Ad-TACI-Igtreated mice (Figure 10C). Thus in this mouse strain, unlike the NZB/W strain, plasma cells in both the spleen and the bone marrow were depleted by Ad-TACI-Ig, but not by Ad-BAFF-R-Ig. Treatment of NZM2410 mice with TACI-Ig protein similarly depleted both splenic and bone marrow plasma cells, but BAFF-R-Ig had no effect on these plasma cells (data not shown).

To determine whether TACI-Ig or BAFF-R-Ig prevent the emergence of IgG plasma cells during a T cell-dependent response,
Ad-BAFF-R-Ig, decreased serum levels of IgM and IgG by $50 \%$, and only of Ad-TACI-Ig-treated mice. The decrease in plasma cells was

Table 2

Absolute number of spleen cells

\begin{tabular}{|c|c|c|c|c|}
\hline Subset & $\begin{array}{c}\text { 16-week-old } \\
\text { NZB/WA } \\
(n=5)\end{array}$ & $\begin{array}{c}\text { 36-week-old } \\
\text { NZB/W } \\
(n=7)\end{array}$ & $\begin{array}{c}\text { 36-week-old } \\
\text { Ad-BAFF-R-Ig/ } \\
\text { CTLA4Ig }(n=5)\end{array}$ & $\begin{array}{l}\text { 36-week-old } \\
\text { Ad-BAFF-R-Ig } \\
(n=4)\end{array}$ \\
\hline Total cell number $\left(\times 10^{7}\right)$ & $9.0 \pm 1.0$ & $13.6 \pm 2.5$ & $4.4 \pm 1.9^{B}$ & $6.6 \pm 3.2^{c}$ \\
\hline CD19 $\times 10^{7}$ & $2.8 \pm 0.1$ & $6.8 \pm 1.8$ & $1.6 \pm 0.9^{B}$ & $1.3 \pm 0.9^{B}$ \\
\hline CD19/CD69 × $10^{6}$ & $1.9 \pm 3.0$ & $8.6 \pm 2.6$ & $1.0 \pm 0.5^{D}$ & $0.6 \pm 0.1^{D}$ \\
\hline Follicular $\times 10^{7}$ & $2.2 \pm 0.1$ & $5.5 \pm 2.1$ & $1.3 \pm 1.1^{D}$ & $1.0 \pm 0.8^{D}$ \\
\hline $\mathrm{T} 1 \times 10^{6}$ & $2.8 \pm 0.8$ & $4.3 \pm 0.4$ & $2.9 \pm 2.5$ & $2.2 \pm 1.5$ \\
\hline Marginal zone × $10^{6}$ & $4.0 \pm 0.7$ & $3.7 \pm 1.1$ & $0.4 \pm 0.2^{B}$ & $0.5 \pm 0.4^{B}$ \\
\hline$B 1 \times 10^{6}$ & ND & $5.4 \pm 2.1$ & $4.4 \pm 2.8$ & ND \\
\hline IgM-//gD- $\times 10^{6}$ (switched) & $1.9 \pm 0.4$ & $10.1 \pm 5.0$ & $3.1 \pm 0.9 \mathrm{D}$ & $2.9 \pm 1.0^{\mathrm{D}}$ \\
\hline $\operatorname{CD} 4 \times 10^{7}$ & $2.3 \pm 0.3$ & $3.4 \pm 0.6$ & $2.6 \pm 1.7$ & $1.4 \pm 0.2^{B}$ \\
\hline $\mathrm{CD} 4 / \mathrm{CD} 69 \times 10^{6}$ & $1.4 \pm 0.2$ & $12.9 \pm 3.6$ & $1.6 \pm 0.3^{B}$ & $2.8 \pm 0.2^{c}$ \\
\hline $\mathrm{CD}^{2} / \mathrm{CD}_{4}+/ \mathrm{CD}^{2} 2 \mathrm{~L}^{-}$(memory) $\times 10^{7}$ & $0.6 \pm 0.2$ & $2.5 \pm 0.5$ & $0.9 \pm 0.7^{B}$ & $0.6 \pm 0.4^{B}$ \\
\hline CD4/CD44-/CD62L+ (naive) $\times 10^{7}$ & $0.9 \pm 0.3$ & $0.5 \pm 0.3$ & $1.4 \pm 0.8$ & $0.5 \pm 0.2$ \\
\hline $\mathrm{CD} 8 \times 10^{7}$ & $0.8 \pm 0.1$ & $0.6 \pm 0.13$ & $1.3 \pm 0.5$ & $0.7 \pm 0.1$ \\
\hline CD11c/CD11b $\times 10^{7}$ & $1.3 \pm 0.2$ & $2.6 \pm 1.0$ & $0.6 \pm 0.4^{B}$ & $0.6 \pm 0.5^{c}$ \\
\hline
\end{tabular}

Values are mean \pm SD. APreviously published in ref. $10 .{ }^{\mathrm{B}} P<0.01,{ }^{\mathrm{C}} P<0.02$, $\mathrm{D} P<0.05$ versus agematched controls. 

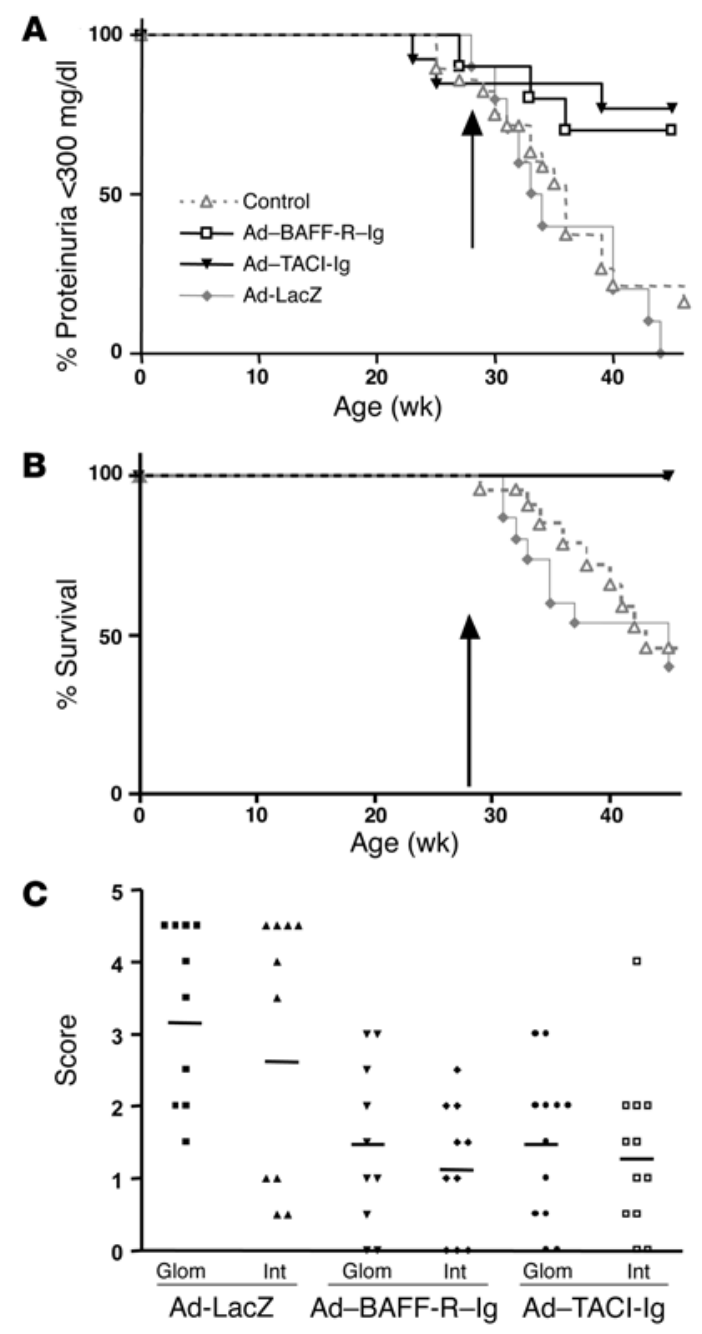

NZM2410 mice were treated with a single dose of adenovirus expressing either fusion protein and immunized 1 week later with 4-hydroxy-3-nitrophenylacetyl (NP) coupled to keyhole limpet hemocyanin (NP-KLH). As expected, the IgM response to NP was completely suppressed only in Ad-TACI-Ig-treated mice (Figure 12A). However, antibodies of the IgG isotype, including IgG1, $\operatorname{IgG} 2 \mathrm{a}$, and IgG2b (data not shown), were detected in the serum of both Ad-TACI-Ig- and Ad-BAFF-R-Ig-treated mice starting 2 weeks after immunization. When these mice were examined at day 21 after immunization there was no difference in the titers of highaffinity $\operatorname{IgG}$ antibodies to NP [NP(3)-BSA] between control mice and either of the treated groups (Figure 12B). As had been observed in unimmunized mice (Figure 10), at 4 weeks after immunization the frequency of B cells producing total IgG measured by ELISpot

\section{Figure 6}

$\operatorname{lgM}, \lg G 1$, and IgG2a anti-oxazolone titers. Mean \pm SD anti-oxazolone (Anti-ox) titers in Ad-LacZ-, Ad-BAFF-R-Ig-, and Ad-TACl-Igtreated NZB/W F1 mice ( $n=8$ per group). Titers were measured 1 and 2 weeks after immunization (IgM: $P<0.003, \mathrm{Ad}-\mathrm{TACl}-\mathrm{Ig}$ versus Ad-LacZ and Ad-BAFF-R-Ig; IgG1 and IgG2a: $P<0.006$, Ad-BAFF$\mathrm{R}-\mathrm{Ig}$ and $\mathrm{Ad}-\mathrm{TACl}-\mathrm{Ig}$ versus Ad-LacZ; $P<0.004$, Ad-BAFF-R-Ig and $\mathrm{Ad}-\mathrm{TACl}-\mathrm{lg}$ versus naive). Asterisks indicate significant differences.

\section{Figure 5}

Ad-BAFF-R-Ig/CTLA4Ig induces disease remission. Kaplan-Meier plots of proteinuria-free $(A)$ and overall survival $(B)$ in NZB/W F1 mice treated at age 28-30 weeks (arrows) with Ad-BAFF-R-Ig, Ad-TACIIg, or Ad-LacZ, each given with 6 doses of CTLA4Ig, and untreated controls. Ad-BAFF-R-Ig or Ad-TACl-Ig treatment delayed proteinuria (A; $P<0.0032$, Ad-BAFF-R-Ig/CTLA4lg versus Ad-LacZ/CTLA4Ig; $P<0.0006$, Ad-TACl-Ig/CTLA4lg versus Ad-LacZ/CTLA4Ig) and prolonged survival (B; $P<0.0035$, Ad-BAFF-R-Ig/CTLA4Ig and AdTACl-Ig/CTLA4Ig versus Ad-LacZ/CTLA4Ig). (C) Histologic analysis of kidneys showed significantly less glomerular damage (Glom; $P<0.01$ ) and interstitial inflammation (Int; $P<0.05$ ) in the kidneys of both sets of treated mice compared with Ad-LacZ controls. A score of 4.5 indicates death of an animal due to uremia. Horizontal lines denote the mean score for each group.

was markedly decreased in the spleens and bone marrow only of Ad-TACI-Ig-treated mice (data not shown).

\section{Discussion}

The TNF-like molecule BAFF (also known as B lymphocyte stimulator $[\mathrm{BLyS}]$ ) is a key $\mathrm{B}$ cell survival factor that binds to 3 receptors - TACI, BCMA, and BAFF-R - that are expressed on the B cell surface at different times throughout $B$ cell ontogeny (19). The homologous molecule APRIL binds only TACI and BCMA $(4,20)$. Variability in the relative expression of the 3 BAFF receptors on $\mathrm{B}$ cells determines whether the B cells will compete for BAFF or APRIL and helps to regulate the survival and homeostasis of the multiple B cell subcompartments (5). Antagonism of BAFF and APRIL is a promising therapeutic approach for B cell-mediated autoimmunity, and both selective (BAFF alone) and nonselective (BAFF and APRIL) blockers are being developed for human use (12). It is currently not known whether there is a therapeutic advantage or an increased toxicity of the nonselective antagonists.

We have therefore analyzed the differences between selective blockade of BAFF alone and blockade of both BAFF and APRIL in an autoimmune disease model. We administered a single dose of adenovirus expressing BAFF-R-Ig to 18- to 20-week-old NZB/W F1 mice either with or without 6 doses of CTLA4Ig. The results were compared with the effects of a similar protocol using Ad-TACI-Ig (10). Ad-BAFF-R-Ig treatment significantly delayed the onset of proteinuria and, like Ad-TACI-Ig, prolonged the life span of NZB/W F1 mice. This effect was very robust, with prolongation of life span by 6 months. As had been observed with Ad-TACI-Ig, the addition of CTLA4Ig to Ad-BAFF-R-Ig did not significantly prolong life compared with Ad-BAFF-R-Ig alone.

Despite minor differences in the pharmacokinetics of fusion protein expression, many of the immunologic effects of the $2 \mathrm{BAFF}$ blocking reagents were similar. Both reagents spared $\mathrm{T} 1$ cells and depleted marginal zone B cells and follicular B cells to the same degree. Given the known turnover rate of these cell populations (5)

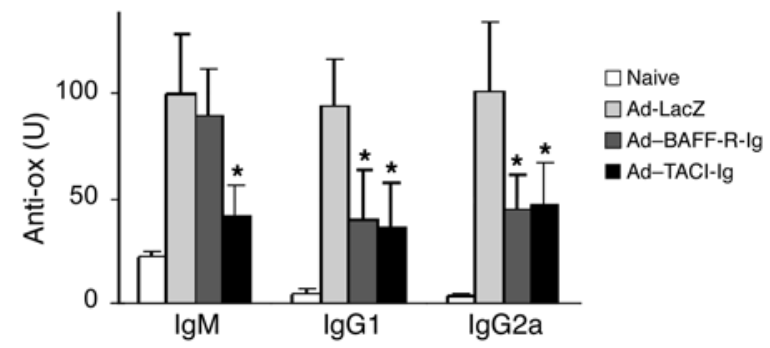




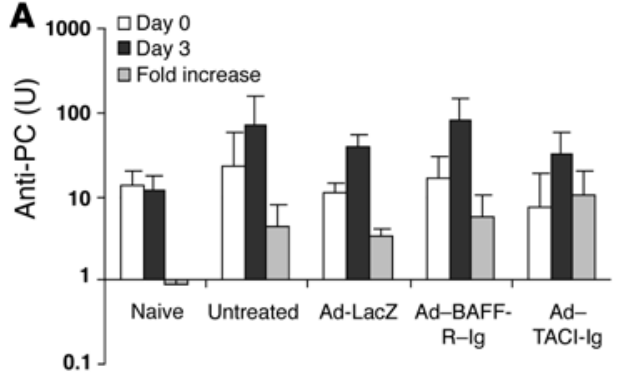

C
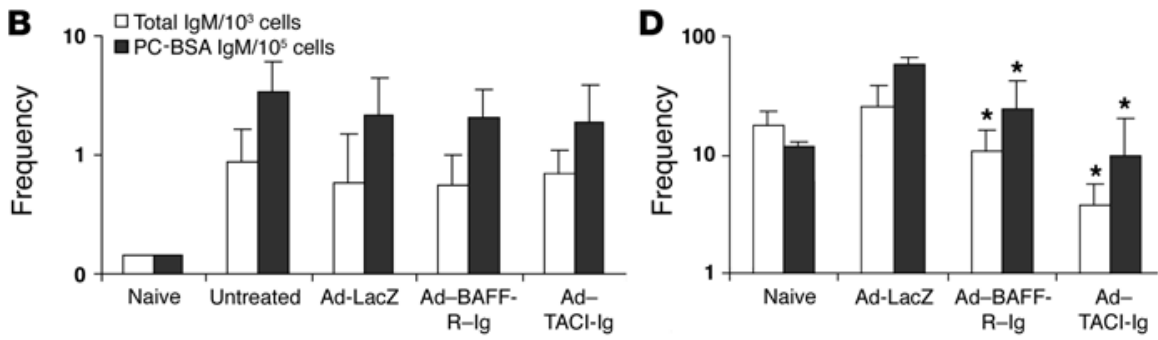

\section{Figure 7}

Ad-BAFF-R-Ig and Ad-TACl-Ig inhibit the splenic response to bacteria but have no effect on peritoneal B1 cells. Anti-PC response in NZB/W F1 mice following i.p. or i.v. immunization with heat-killed streptococci. Following i.p. injection, serum IgM anti-PC antibody levels (A) and IgM ELISpot analysis of the peritoneal cells (B) showed no difference between treatment groups and controls. Following i.v. injection, serum IgM anti-PC antibody levels failed to increase following immunization in treated mice (C; $P<0.01$, Ad-BAFF-R-Ig and Ad-TACl-Ig versus Ad-LacZ), and ELISpot analysis of the spleen cells (D) showed that both Ad-BAFF-R-Ig and Ad-TACl-Ig suppressed the IgM anti-PC response $(P<0.01$, $\mathrm{Ad}-\mathrm{TACl}-\mathrm{Ig}$ versus $\mathrm{Ad}$-LacZ for both IgM and anti-PC; $P<0.04$, Ad-BAFF-R-Ig versus Ad-LacZ for both IgM and anti-PC). Asterisks indicate significant differences. and the depletion observed within 1 week of fusion protein administration, our present findings confirm the dominant role of BAFF/ BAFF-R interactions for survival of marginal zone and follicular populations, even late in the disease process, in autoimmune mice.

Despite the prolonged loss of B cells associated with both reagents, IgG anti-DNA antibodies appeared in the serum after only a short delay and deposited in the kidney. It has previously been reported that a 4-week course of BAFF-R-Ig prevents the emergence of IgG anti-DNA antibodies in NZB/W mice for many months (9). This is of interest because it has been suggested that naive high-affinity autoreactive $B$ cells fail to compete for sur- vival in a BAFF-deficient environment $(21,22)$. Thus depletion of BAFF might alter selection of autoreactive $B$ cells into the naive repertoire. On the other hand, because BAFF depletion reduces B cell surface expression of complement receptors (23), it is theoretically possible that autoreactive $B$ cells that would be deleted or anergized by a strong BCR signal would be spared. These conflicting hypotheses remain to be addressed in autoimmune models. Despite BAFF blockade using BAFF-R-Ig, IgG anti-double-stranded DNA (anti-dsDNA) antibodies reproducibly arose with only a short delay in more than 30 mice studied using both ELISA and ELISpot assay. Our results show that BAFF-R-Ig and TACI-Ig had
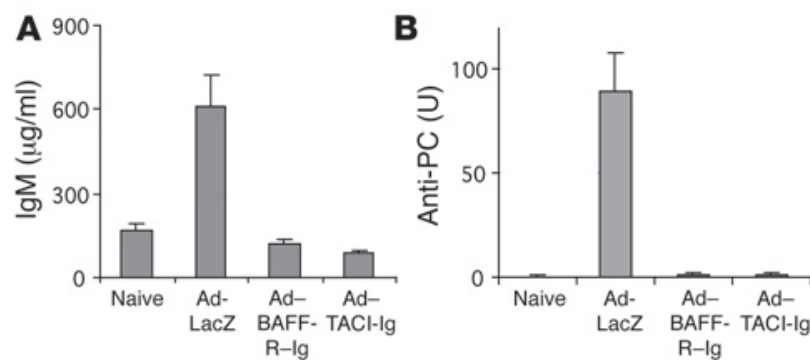

C

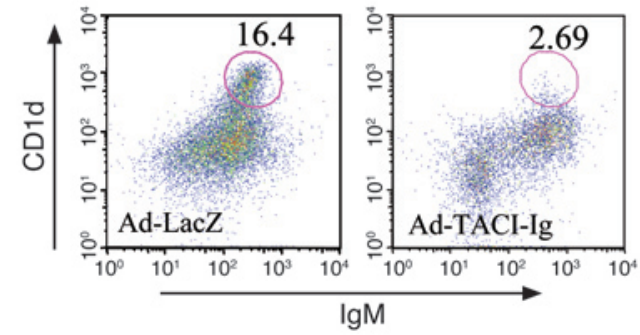

D

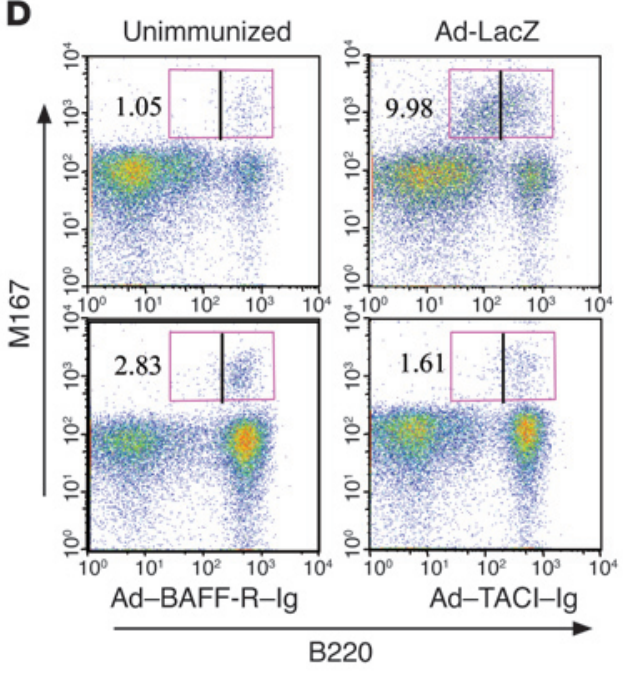

Figure 8

Both Ad-BAFF-R-Ig and Ad-TACI-Ig deplete marginal zone B cells in M167 mice. Mice were immunized with heat-killed streptococci after pretreatment with Ad-BAFF-R-Ig or Ad-TACl-Ig. Following immunization, an increase in serum IgM levels (A) and IgM anti-PC antibodies (B) was seen only in the control group $(P<0.01$, Ad-LacZ versus Ad-BAFF-R-Ig and Ad-TACl-Ig for both IgM and anti-PC). (C) FACS analysis

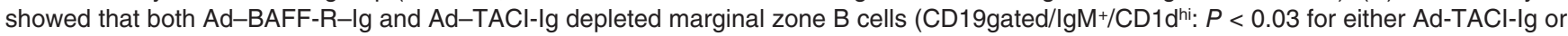
Ad-BAFF-R-Ig treatment versus untreated controls). Similar depletion was observed in treated mice that were not immunized (not shown). (D) Both treatments prevented the expansion of $\mathrm{M} 167^{+} \mathrm{B} 220^{10}$ plasmablasts (left half of red box, $P<0.01$ for either Ad-TACI-Ig or Ad-BAFF-R-Ig treatment versus untreated controls; numbers represent total number of $\mathrm{M} 167^{+}$cells). Representative FACS plots are shown. 

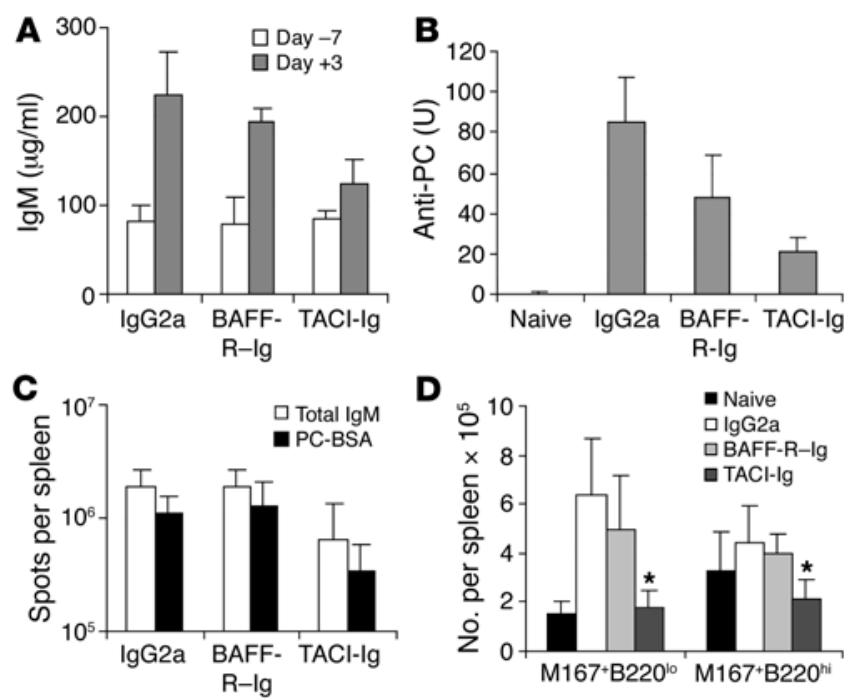

a similar effect on the anti-DNA response, with a delay equivalent to the time that the fusion protein was expressed in the serum. The nature of the anti-DNA antibodies that emerged over time was further studied in TACI-Ig-treated mice. In these mice, spontaneous germinal centers still formed, and IgG-secreting plasma cells appeared in the bone marrow. Furthermore, the relative affinity of the antibodies for DNA was not different from that of controls, and somatic mutations occurred at an equivalent rate, indicating that autoreactive $B$ cells received $T$ cell help, most likely in the germinal center (10). In both BAFF-R-Ig- and TACI-Ig-treated mice, deposition of antibodies in the glomeruli was observed well before disease onset. This suggests that the effect of BAFF blockade is not simply due to prevention of autoantibody formation.

$\mathrm{B}$ cells produce soluble mediators involved in the organization of lymphoid tissues, and their absence results in shrinkage of the spleen and lymph nodes $(24,25)$. B cells also function as antigenpresenting cells that participate in cycles of autoantigen presentation to autoreactive T cells (26). Because of the small spleen size and the decreased number of activated $B$ cells in treated mice, the total number of activated and memory $\mathrm{T}$ cells did not increase over time. BAFF-R-Ig and TACI-Ig were similar with respect to their effects on $\mathrm{T}$ cell numbers and activation state, both early and late in disease progression. This effect may be explained by B cell depletion alone. It has also been suggested that BAFF-R mediates costimulation of T cells (27), and that BAFF blockade might therefore directly inhibit $\mathrm{T}$ cell activation. Further work is needed to define the role of BAFF-R as a $\mathrm{T}$ cell costimulatory molecule in vivo. The small spleen size also resulted in a decrease in the total number of CD11c/CD11b-positive dendritic cells. These cells can

\section{Figure 10}

TACI-Ig depletes plasma cells in NZM2410 mice. NZM2410 mice (14 weeks old) were injected with a single dose of $\mathrm{Ad}-\mathrm{TACl}-\mathrm{Ig}$, Ad-BAFFR-Ig, or Ad-LacZ. Serum IgM (A) and IgG (B) were decreased only in the Ad-TACl-Ig-treated group $(P<0.004$ for both IgM and IgG, ANOVA). (C) ELISpot analysis of spleen and bone marrow cells 4 weeks after treatment showed a significant reduction in the frequency of IgM and IgG producing cells in the spleen and bone marrow only in the Ad-TACl-Ig-treated group $(P<0.003, \mathrm{Ad}-\mathrm{TACl}-\mathrm{Ig}$ versus Ad-LacZ for both IgM and IgG).

\section{Figure 9}

TACl-Ig protein inhibits the response of marginal zone B cells. Anti$\mathrm{PC}$ response following i.v. immunization of M167 mice with heat-killed streptococci after pretreatment with BAFF-R-Ig or TACI-Ig protein as described in Methods. Only the TACl-Ig-treated group manifested a significant reduction in serum IgM levels $(\mathbf{A} ; P<0.01$ versus IgG2a and BAFF-R-Ig), serum IgM anti-PC antibodies (B; $P<0.02$ versus $\operatorname{lgG} 2 \mathrm{a})$, the number of IgM- and anti-PC antibody-secreting cells in spleen (C; $P<0.02$ versus IgG2a and BAFF-R-Ig), and the number of M167+B220lo B cells (D; $P<0.03$ versus BAFF-R-Ig; $P<0.02$ versus IgG2a) and plasmablasts quantitated as in Figure $8(P<0.02$ versus BAFF-R-Ig and IgG2a). Asterisks indicate significant differences.

migrate to the kidneys of NZB/W F1 mice and secrete chemokines that attract inflammatory cells into the kidney (28). Thus the delay in disease onset was due to the marked reduction in the number of effector cells available to secrete inflammatory mediators and to respond to the deposition of immune complexes in the kidney.

The most striking difference between BAFF-R-Ig and TACI-Ig treatment was the profound effect only of TACI-Ig on the survival of certain subgroups of plasma cells. NZB/W mice accumulate large numbers of IgM-secreting plasma cells in their spleens, which include both short-lived and long-lived cells (29). Administration of TACI-Ig to NZB/W F1 mice resulted in depletion of these IgMsecreting plasma cells from the spleen red pulp and a reduction in serum levels of IgM antibodies that lasted for months after admin-
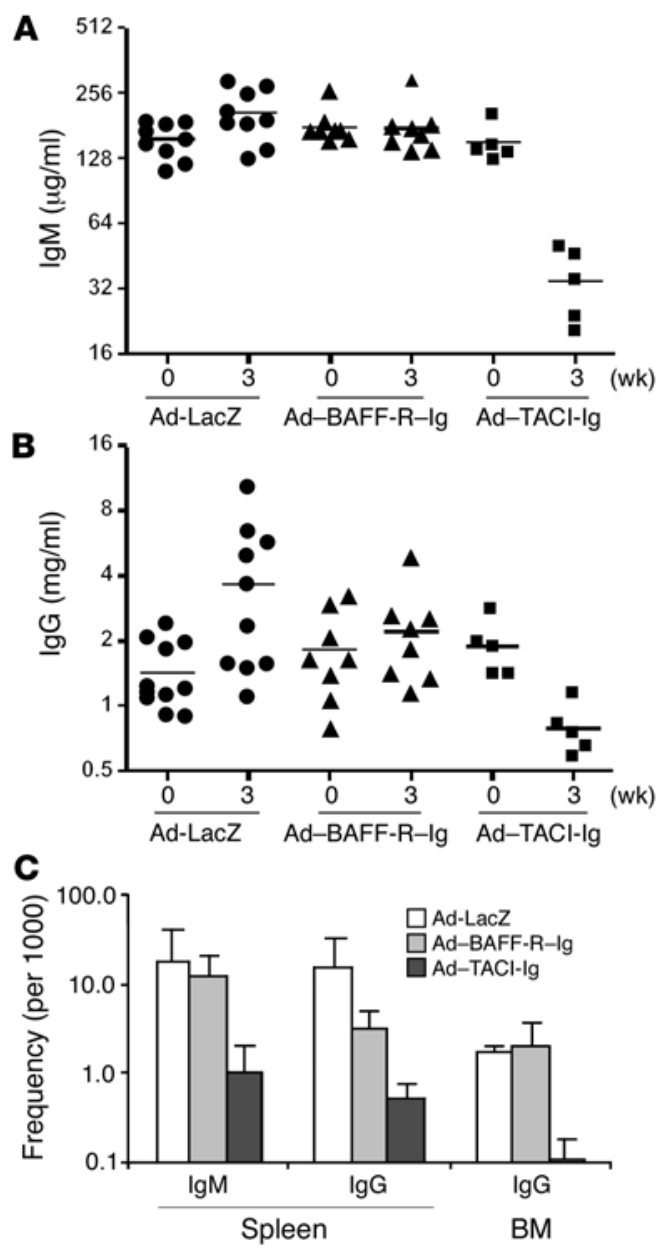
NZB/W F1
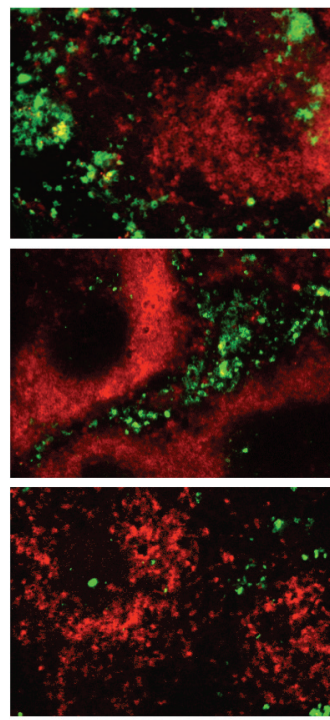

$\lg \mathrm{M} / \lg \mathrm{D}$

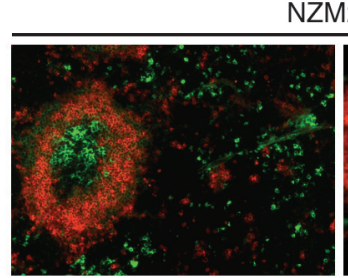

NZM2410

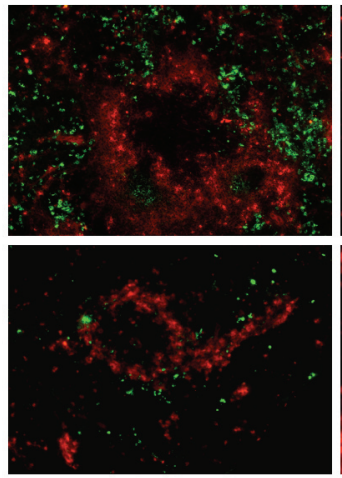

$\lg \mathrm{M} / \lg \mathrm{D}$
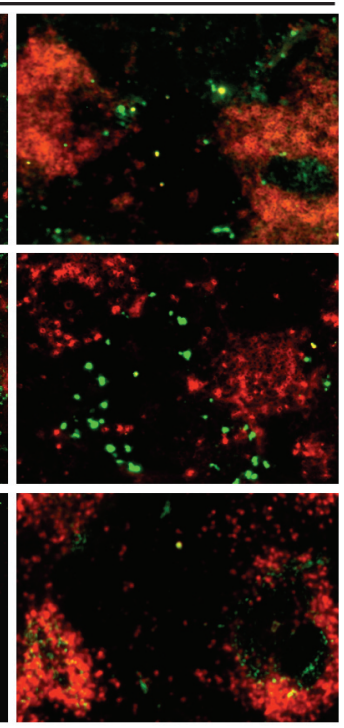

$\lg G / \lg D$

\section{Figure 11}

Depletion of splenic plasma cells by Ad-TACI-Ig. Immunohistochemistry of spleens stained with anti-IgM (green) and -IgD (red) from NZB/W F1 mice treated with Ad-LacZ (top panels), Ad-BAFF-R-Ig (middle panels), and $\mathrm{Ad}-\mathrm{TACl}-\mathrm{Ig}$ (bottom panels). IgM-containing plasma cells were abundant in the red pulp of control and $\mathrm{Ad}-$ BAFF-R-Ig-treated mice but were smaller and less frequent in Ad-TACl-Ig-treated mice. Anti-IgD and anti-IgM or anti-IgG staining of spleens from NZM2410 mice that were treated 1 week previously with Ad-LacZ, Ad-BAFFR-Ig, Ad-TACl-Ig (bottom center panel), or left untreated (bottom right panel) similarly shows that both IgM and IgG plasma cells were depleted only in the spleens of Ad-TACl-lg-treated mice. Magnification, $\times 10$. istration, suggesting either that TACI-Ig induced a long-lasting change in the spleen microenvironment or that the cells took a long time to reaccumulate. In contrast, BAFF-R-Ig had no effect on these plasma cells. TACI-Ig, but not BAFF-R-Ig, also inhibited the emerging IgM anti-DNA response and the IgM response to a $\mathrm{T}$ cell-dependent antigen, indicating either that $\mathrm{B}$ cells were diverted from the extrafollicular pathway or that newly formed extrafollicular plasma cells were unable to survive in the BAFF- and APRIL-deficient spleen. This was not simply due to the changes in spleen size that resulted from $B$ cell depletion, because the $B$ cell-depleting effects of BAFF-R-Ig and TACI-Ig were the same. In NZM2410 mice the differences between TACI-Ig and BAFF-R-Ig were even more striking. In this strain TACI-Ig depleted both spleen and bone marrow plasma cells, resulting in a decrease in serum levels of both $\operatorname{IgM}$ and IgG, whereas BAFF-R-Ig had no effect on serum Igs or Ig-secreting cells despite having similar B cell-depleting effects. A similar difference between BAFF-R-Ig and BCMA-Ig has been noted with respect to bone marrow plasma cells in normal mice (30). It remains to be determined whether this difference between TACI-Ig and BAFF-R-Ig will influence disease outcome in the NZM2410 strain.

We next examined the function of the various B cell subcompartments. Neither BAFF-R-Ig nor TACI-Ig altered the number of B1 cells in the spleen or peritoneum. The role of BAFF and APRIL in $B 1$ cell survival is still controversial, even in normal mice. Some - but not all - BAFF transgenic strains have increased numbers of B1 cells $(8,31,32)$, and TACI-Ig transgenic mice lose B1 cells with age (33). Furthermore, APRIL transgenic mice develop large numbers of B1 cells and B1 lymphomas with age, suggesting that

\section{Figure 12}

TACl-Ig and BAFF-R-Ig have similar effects on the germinal center response. Following NP-KLH immunization in NZM2410 mice, the serum $\mathrm{IgM}$ anti-NP response was significantly reduced only in the Ad-TACl-Igtreated mice $[\mathbf{A}$; NP(30)-BSA, $P<0.005, \mathrm{Ad}-\mathrm{TACl}-\mathrm{Ig}$ versus Ad-LacZ and Ad-BAFF-R-Ig], while the IgG anti-NP response [B; NP(3)-BSA] was variable and not significantly different between treated mice and controls. Results are shown at week 2 for IgM and week 3 for lgG.
APRIL plays a role in the homeostasis of this compartment (34). In contrast to findings in normal mice (35), immunization studies revealed that neither BAFF-R-Ig nor TACI-Ig blocked the response of peritoneal NZB/W B1 cells to streptococci administered i.p. However, high doses of both fusion proteins completely inhibited the $T$ cell-independent response to i.v. administered streptococci, a response that is derived from both marginal zone B cells and splenic B1 B cells. This suggests that despite the normal numbers of $\mathrm{B} 1$ cells in the spleens of treated mice, BAFF is required for the generation of a functional immune response by these cells. These findings are consistent with previous data that show that TACI is required for T cell-independent responses (36) and that the relevant ligand is $\operatorname{BAFF}(37,38)$. The absence of change in either the size or function of the peritoneal B1 compartment in NZB/W F1 mice may be due to the presence of other supporting cells or

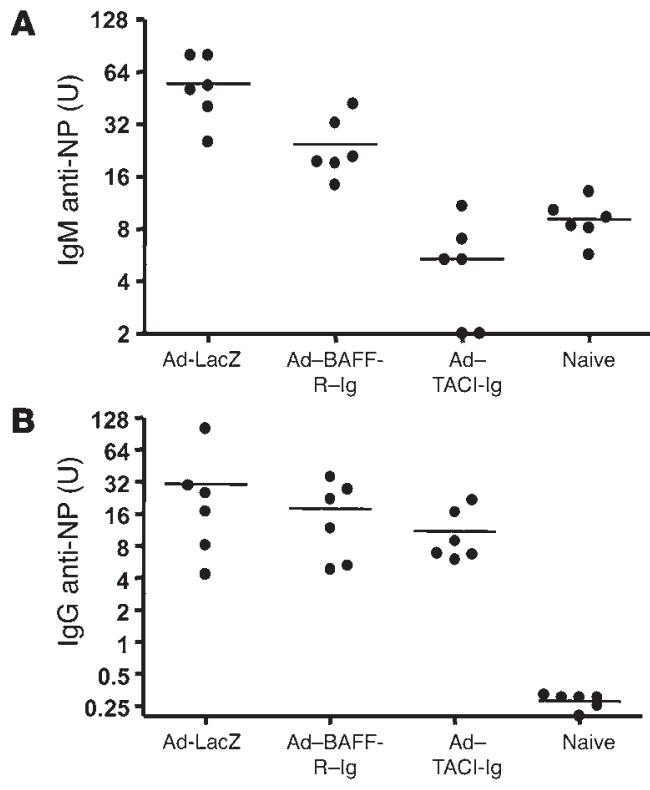


inflammatory cytokines in the NZB/W peritoneal microenvironment. Additionally, there may be functional differences between B1 cells in the spleen and the peritoneum (39) that account for the observed differences in BAFF dependence between the 2 sites.

To further study the effect of BAFF blockade, specifically on the function of marginal zone B cells, we performed immunization studies in M167 transgenic mice in which the immune response to bacteria preferentially derives from $\mathrm{M} 67^{+}$cells in the marginal zone. High doses of BAFF-R-Ig and TACI-Ig caused profound depletion of marginal zone $\mathrm{B}$ cells that was evident within 10 days of treatment with either fusion protein. It has previously been shown that TACI-Ig also affects survival of marginal zone B cellderived plasmablasts in the spleen (35). When lower, equal doses of TACI-Ig and BAFF-R-Ig were administered as protein to M167 mice, marginal zone B cell depletion was incomplete. Under these circumstances, TACI-Ig had a more significant effect on the expansion or survival of $\mathrm{Id}^{+} \mathrm{B}$ cells and the emergence of plasmablasts than did BAFF-R-Ig. Similar results were observed in NZB/W mice, suggesting that APRIL can compensate for BAFF deficiency with respect to mediating marginal zone-derived extrafollicular plasmablast survival in the spleen.

The IgG response to a T cell-dependent antigen was only partially inhibited by both reagents. While BAFF deficiency is known to result in smaller and shorter-lived germinal centers and lower antibody titers in response to immunization with $\mathrm{T}$ cell-dependent antigens, it does not prevent class switching or affinity maturation in normal mice $(40,41)$. Our previous findings show that this also appears to be the case in autoimmune mice (10). Recent analysis of BAFF-R expression in human germinal centers has shown that BAFF-R is expressed early in germinal center development and in the plasma cell precursors, but that as plasma cells develop, their expansion is maintained by TACI and BCMA (14). However, since APRIL is not expressed within the germinal centers (14), these are predominantly dependent on BAFF. Our data is consistent with this finding, as BAFF-R-Ig and TACI-Ig had indistinguishable effects on IgG anti-DNA antibody responses (10) and IgG antioxazolone responses in NZB/W mice. This was also apparent in the NZM2410 strain, which has large numbers of plasma cells in the red pulp of the spleen. Young NZM2410 mice have very few plasma cells in the bone marrow (18), but we have found that plasma cells did accumulate in the bone marrow of these mice as they aged. Despite the profound loss of red pulp IgG-secreting plasma cells in TACI-Ig-treated NZM2410 mice, these mice mounted a measurable high-affinity IgG response to the T cell-dependent antigen NP-KLH given with adjuvant, which was no different from that in BAFF-R-Ig-treated mice or controls. Our experiment did not examine the longevity of these newly derived plasmablasts past 28 days, and it remains to be determined whether TACI-Ig alters the survival of the plasma cells that subsequently migrate out of the germinal centers to other sites.

It has recently been reported that BCMA is the predominant $\mathrm{BAFF} / \mathrm{APRIL}$ receptor expressed on bone marrow plasma cells in mice (42). Since mouse BAFF binds only poorly to mouse BCMA (43), APRIL is an important mediator of plasma cell survival in the mouse bone marrow, and these cells can be depleted in normal mice with TACI-Ig. We did not observe any effect of TACI-Ig on IgG-secreting B cells in the bone marrow of NZB/W mice (ref. 10 and $\mathrm{Z}$. Liu, unpublished observations). In contrast, bone marrow plasma cells in the NZM2410 mouse were highly susceptible to TACI-Ig but not to BAFF-R-Ig. These strain differences may reflect differential expression of the various BAFF receptors or the amount of available BAFF and APRIL. However, our previous finding that the combination of CTLA4Ig and TACI-Ig is able to deplete serum IgG in NZB/W mice (10) suggests that there are likely other factors within the bone marrow environment that support plasma cell survival and may account for the observed strain differences.

Of relevance to the use of BAFF blockade as a therapy for autoimmunity, the combination of Ad-BAFF-R-Ig and CTLA4Ig was able to induce remission of established disease when administered at the age of 28-30 weeks. B cell depletion occurred following this late treatment, but the effects on activated $\mathrm{T}$ cells were less than those observed in mice treated at an earlier stage of disease. The precise mechanism by which BAFF blockade induces disease remission remains to be determined, but may include a direct effect on the kidney itself since BAFF (but not APRIL) is expressed at high levels in the inflamed kidney at the onset of clinical nephritis (L. Schiffer and A. Davidson, unpublished observations).

Our findings are relevant to the application of BAFF blockade to the treatment of human disease. For diseases in which B cell depletion is the desired outcome, both selective and nonselective BAFF blockade should be effective. In contrast, TACI-Ig may be the agent of choice for diseases mediated by short-lived or extrafollicular plasma cells and those in which the predominant autoantibody specificity is of the IgM class. Furthermore, TACI-Ig may be able to deplete long-lived plasma cells in some individuals. This is clinically of great importance, given that at least part of the autoantibody pool may derive from these cells (25), and may also be relevant for the treatment of $\mathrm{B}$ cell malignancies. Identification of the BAFF and APRIL receptors present on malignant $B$ cells could direct therapy with BAFF and/or APRIL blockade. Differences between mice and humans might occur because mouse BAFF binds poorly to BCMA, whereas human BAFF binds to BCMA well. It is clear that a short course of BAFF blockade has long-lasting effects, suggesting that some treatment goals might be achieved by intermittent courses of therapy. However, resting memory B cells do not appear to be susceptible to BAFF blockade (10). Further work remains to be done to analyze the in vivo effects of BAFF blockade on reactivation of memory $B$ cells and to determine whether continuous therapy is required to suppress the production of autoantibodies derived from turnover of the memory compartment. It has been proposed that plasma cells are continuously generated from polyclonal activation of memory cells (44). The contribution of such a mechanism to autoreactivity and the effect of BAFF blockade on this phenomenon have not been explored.

Use of either TACI-Ig or BAFF-R-Ig may cause immunosuppression with respect to responses to blood-borne encapsulated microorganisms to which marginal zone B cells and B1 cells constitute the first line of defense. Our data suggest that TACI-Ig may be more potent with respect to this side effect. It has recently been reported that TACI-deficient individuals are $\operatorname{IgM}$ deficient and suffer from an immunodeficiency with recurrent infections due to encapsulated bacteria (45). Unlike other B cell-depleting agents, BAFF blockade only partially modulates germinal center responses; affinity maturation and class switching occur in germinal centers despite continued profound B cell depletion (40). Thus protective IgG responses to previously unencountered $\mathrm{T}$ cell-dependent antigens may remain intact. However, the preferential loss of $\operatorname{IgM}$ in TACI-Ig-treated mice may result in a more deleterious impact on early $\mathrm{T}$ cell-dependent immune responses to infectious organisms mediated by neutralizing IgM antibodies. As drugs that block BAFF 
and APRIL progress to human clinical trials in a variety of diseases, translational studies in humans should address the potential differences in the effects of selective versus nonselective BAFF blockade on $B$ cell survival within the various microenvironments and on adverse events caused by immunosuppression.

\section{Methods}

Generation of adenoviruses expressing fully murine BAFF-R-Ig and TACI-Ig. The extracellular domain of murine BAFF-R (aa 2-76) was cloned from NZB/W F1 spleen cDNA by RT-PCR and fused to a human $\beta 2$ microglobulin signal sequence and to the $\mathrm{CH} 2-\mathrm{CH} 3$ domains of murine IgG2a, mutated at the $\mathrm{Fc}$ receptor binding site. The construct was expressed in Adeno-X adenovirus according to the manufacturer's instructions (Clontech Laboratories Inc.). Expression of BAFF-R-Ig was confirmed by immunoprecipitation of serum from SCID mice injected with $1 \times 10^{9}$ PFU Ad-BAFF-R-Ig and by BAFF-Rspecific ELISA using anti-BAFF-R antibody (kind gift of S. Kalled, Biogen Inc., Cambridge, Massachusetts, USA) to coat the plates and anti-IgG2a for detection. We also reengineered our previously described Ad-TACI-Ig virus (10) by replacing the trypsin signal peptide with the human $\beta 2$ microglobulin signal peptide, resulting in a 5 -fold improvement in expression of TACI-Ig fusion protein in the serum of SCID mice. The $\beta 2$ microglobulin TACI-Ig virus was used for all the experiments described in this manuscript.

Treatment of NZB/W F1 mice. NZB/NZW F1 females were purchased from The Jackson Laboratory. Mice were tested at 18 weeks of age for IgG antidsDNA antibodies and were distributed into matched treatment groups depending on the antibody titer just prior to treatment with Ad-BAFF$\mathrm{R}$-Ig or $\beta$-galactosidase adenovirus (Ad-LacZ; $1 \times 10^{9} \mathrm{pfu} ; n=10$ per group). Treatment was also combined with CTLA4Ig (46): 6 doses of $100 \mu \mathrm{g}$ administered over a 2 -week period $(n=10)$. Comparisons were performed with 10 mice that received CTLA4Ig alone and 20 untreated controls. Mice were bled, and their urine was tested for proteinuria by dipstick (Multistick; Fisher Scientific International), every 2 weeks. Mice were followed until death. All experiments using animals were carried out according to protocols that were reviewed and approved by the Institutional Animal Care and Use Committees of Columbia University and Albert Einstein College of Medicine.

Serum IgM, IgG, and anti-dsDNA antibodies. Measurement of serum IgM and IgG levels and of anti-dsDNA antibodies was performed as previously described (46).

Anti-dsDNA ELISpot assay. Spleens were harvested from 4-5 mice in each group at 35-37 weeks of age by survival splenectomy as previously described (46). Spleens were weighed, and total cell count was performed. ELISpot assays for total Ig-secreting cells and for anti-dsDNA-secreting B cells were performed as previously described (46).

Flow cytometry analysis of spleens. Spleen cells were analyzed for lymphocyte markers as previously described (10).

Remission induction studies. Groups of $10 \mathrm{NZB/W}$ F1 mice were treated with Ad-LacZ/CTLA4Ig, Ad-BAFF-R-Ig/CTLA4Ig, or Ad-TACI-Ig/ CTLA4Ig at 28-30 weeks of age. We used the combination treatment because we have previously shown efficacy with Ad-TACI-Ig/CTLA4Ig. Mice were bled, and their urine was tested for proteinuria by dipstick, every 2 weeks. Serum IgM, IgG, and anti-DNA antibodies were measured as described above. The mice were sacrificed 15 weeks later, and their spleens were harvested for B cell analysis by FACS and ELISpot. Kidneys obtained at sacrifice were stained with $\mathrm{H} \& \mathrm{E}$ and scored by an independent observer using a $0-4$ score for glomerular damage and interstitial and vascular inflammation as previously described (47).

Immunization of NZB/W F1 mice with the T cell-dependent antigen oxazolone. Groups of $5 \mathrm{NZB} / \mathrm{W}$ F1 mice were immunized with $750 \mu \mathrm{g}$ oxazolone (Sigma-Aldrich) by skin paint at 26 weeks of age, 8 weeks after treatment with Ad-LacZ, Ad-BAFF-R-Ig, or Ad-TACI-Ig. Mice were bled at weekly intervals, and anti-oxazolone antibodies were measured by ELISA as previously described (48).

Immunization of $N Z B / W F 1$ mice with the $T$ cell-independent antigen heat-killed R36A streptococci. Five to ten 20-week-old NZB/W F1 mice were injected i.v. with Ad-TACI-Ig, Ad-BAFF-R-Ig, or Ad-LacZ. One week later, mice were immunized with heat-killed, pepsin-treated Streptococcus pneumoniae (strain R36A bacteria, kind gifts of J. Kearney and D. Briles, University of Alabama, Birmingham, Alabama, USA $)$ either i.v. $\left(1 \times 10^{8}\right.$ bacteria/mouse $)$ or i.p. $\left(5 \times 10^{7}\right.$ bacte$\mathrm{ria} /$ mouse). Three days later the mice were sacrificed, and their spleens were harvested for analysis of spleens (i.v. group) and peritoneal cells (i.p. group). Mice were bled at days 0, 7, and 10 relative to adenovirus injection.

PC-specific ELISA and ELISpots. ELISA plates (BD - Falcon) were coated with $10 \mu \mathrm{g} / \mathrm{ml} \mathrm{PC}(10)$-BSA (Biosearch Technologies Inc.) overnight at $4{ }^{\circ} \mathrm{C}$ and blocked for 90 minutes with 5\% FCS and 3\% BSA in PBS at room temperature. Sera were then incubated in serial dilutions for 1 hour at $37^{\circ} \mathrm{C}$ followed by Fab'2 goat anti-mouse IgM diluted 1:4,000 in PBS with $1 \%$ BSA (SouthernBiotech) and ABTS substrate (KPL Inc.). Serial dilutions of a high-titer serum from an immunized mouse were used in each plate to establish a standard curve. For analysis of the frequency of anti-PC antibody-producing B cells, plates were coated with PC(10)-BSA, and ELISpots were performed as described previously for oxazolone-BSA (49).

Immunization studies using anti-PC transgenic mice. M167 mice (kind gift of J. Kenny, NIH, Baltimore, Maryland, USA) were given a single i.v. injection of Ad-TACI-Ig, Ad-BAFF-R-Ig, or Ad-LacZ at 20 weeks of age and immunized with heat-killed, pepsin-treated $S$. pneumoniae i.v. $\left(1 \times 10^{8}\right.$ bacteria/mouse) as described above for NZB/W F1 mice. Separate groups of mice were treated with $500 \mu \mathrm{g}$ purified protein (BAFF-R-Ig, TACI-Ig, or IgG2a) every 2 days for 5 doses. After the fourth dose, mice were immunized with $5 \times 10^{7}$ heat-killed, pepsin-treated $S$. pneumoniae as described above. Spleens were harvested 3 days after immunization and analyzed for anti-PC response using ELISpot as described above. Spleen cells were also analyzed for expression of B220, CD1d, and the M167 idiotype using flow cytometry. For detection of the M167 idiotype, the anti-M167 hybridoma 28-6-20 (kind gift of J. Kenny) (50) was purified from supernatant using Protein G chromatography and labeled with AlexaFluor 647 (Invitrogen Corp.) according to the manufacturer's instructions.

NZM2410 mice. NZM2410 mice were purchased from Taconic. Groups of 6 14-week-old mice were given a single i.v. injection of Ad-TACI-Ig, Ad-BAFF-R-Ig, or Ad-LacZ. Four weeks later mice were sacrificed, and their spleens were processed for B cell analysis by ELISpot and FACS as described above. Bone marrow cells were processed for ELISpot analysis. Serum IgM and IgG levels were measured by ELISA as described above. Separate groups of mice were also treated with $500 \mu \mathrm{g}$ protein (BAFF-R-Ig, TACI-Ig, or IgG2a) twice weekly for 3 weeks and analyzed in the same way. Groups of 6 NZM2410 mice (12 weeks old) were given a single i.v. injection of Ad-TACI-Ig, Ad-BAFF-R-Ig, or Ad-LacZ. One week later, the mice were immunized i.p. with $100 \mu \mathrm{g}$ NP(34)-KLH (Biosearch Technologies Inc.) in complete Freund's adjuvant. Mice were bled weekly. Serum IgM and IgG anti-NP antibodies were detected by ELISA using NP(30)-BSA and $\mathrm{NP}(3)$-BSA as above.

Immunofluorescence staining. Cryosections $(5 \mu \mathrm{m})$ were stained with PE-conjugated anti-B220, FITC-conjugated anti-mouse IgM, PE-conjugated anti-mouse $\mathrm{IgD}$ (BD - Pharmingen), FITC-conjugated anti-mouse IgG1, or FITC-conjugated anti-mouse IgG2a (SouthernBiotech) in 10\% normal rabbit serum/normal goat serum/PBS for 1 hour at room temperature. Images were captured using a digital CCD camera system (Diagnostic Instruments) connected to a Nikon Inc. microscope.

Statistics. Experiments using 5-6 mice per group were repeated at least once. ELISA data for each antigen was normalized to a high-titer mouse assigned an arbitrary level of $100 \mathrm{U}$ and run in serial dilution on each 
plate. Proteinuria and survival data in Figures 2 and 5 were analyzed using Kaplan-Meier curves and log-rank test. Comparisons in Figures 3, 6-9, and 12 and in Tables 1 and 2 were performed using Wilcoxon rank-sum test. Comparisons in Figure 10 were performed using Kruskal-Wallis test. $P$ values less than or equal to 0.05 were considered significant.

\section{Acknowledgments}

This work was supported by grants from the NIH (PO1 AI51392 to A. Davidson, B. Diamond, and S. Porcelli and the Flow Cytometry and Protein Expression and Tetramer Cores of PO1 AI51392) and grants from the New York SLE Foundation and the New York
Arthritis Foundation (to M. Ramanujam). We thank Michael M. Madaio for assistance with renal histology.

Received for publication July 25, 2005, and accepted in revised form December 25, 2005.

Address correspondence to: Anne Davidson, Columbia University Medical Center, Department of Medicine, 1130 St. Nicholas Avenue, Audobon III Building, 9th Floor, Room 917, New York, New York 10032, USA. Phone: (212) 851-4571; Fax: (212) 851-4548; E-mail:ad2247@columbia.edu.
1. Lipsky, P.E. 2001. Systemic lupus erythematosus: an autoimmune disease of B cell hyperactivity. Nat. Immunol. 2:764-766.

2. Cassese, G., et al. 2001. Inflamed kidneys of NZB/W mice are a major site for the homeostasis of plasma cells. Eur. J. Immunol. 31:2726-2732.

3. Takemura, S., et al. 2001. Lymphoid neogenesis in rheumatoid synovitis. J. Immunol. 167:1072-1080.

4. Mackay, F., Schneider, P., Rennert, P., and Browning, J. 2003. BAFF and APRIL: a tutorial on B cell survival. Annu. Rev. Immunol. 21:231-264.

5. Crowley, J.E., Treml, L.S., Stadanlick, J.E., Carpenter, E., and Cancro, M.P. 2005. Homeostatic niche specification among naive and activated B cells: a growing role for the BLyS family of receptors and ligands. Semin. Immunol. 17:193-199.

6. Zhang, J., et al. 2001. Cutting edge: a role for B lymphocyte stimulator in systemic lupus erythematosus. J. Immunol. 166:6-10.

7. Cheema, G.S., Roschke, V., Hilbert, D.M., and Stohl, W. 2001. Elevated serum B lymphocyte stimulator levels in patients with systemic immune-based rheumatic diseases. Arthritis Rheum. 44:1313-1319.

8. Gross, J.A., et al. 2000. TACI and BCMA are receptors for a TNF homologue implicated in B-cell autoimmune disease. Nature. 404:995-999.

9. Kayagaki, N., et al. 2002. BAFF/BLyS receptor 3 binds the B cell survival factor BAFF ligand through a discrete surface loop and promotes processing of NF-kappaB2. Immunity. 17:515-524.

10. Ramanujam, M., et al. 2004. Mechanism of action of transmembrane activator and calcium modulator ligand interactor-Ig in murine systemic lupus erythematosus. J. Immunol. 173:3524-3534.

11. Wang, H., et al. 2001. TACI-ligand interactions are required for $\mathrm{T}$ cell activation and collagen-induced arthritis in mice. Nat. Immunol. 2:632-637.

12. Ramanujam, M., and Davidson, A. 2004. The current status of targeting BAFF/BLyS for autoimmune diseases. Arthritis. Res. Ther. 6:197-202.

13. Avery, D.T., et al. 2003. BAFF selectively enhances the survival of plasmablasts generated from human memory B cells. J. Clin. Invest. 112:286-297. doi:10.1172/JCI200318025.

14. Zhang, X., et al. 2005. BAFF supports human B cell differentiation in the lymphoid follicles through distinct receptors. Int. Immunol. 17:779-788.

15. Pelletier, M., et al. 2003. Comparison of soluble decoy IgG fusion proteins of BAFF-R and BCMA as antagonists for BAFF. J. Biol. Chem. 278:33127-33133.

16. Martin, F., Oliver, A.M., and Kearney, J.F. 2001. Marginal zone and B1 B cells unite in the early response against $\mathrm{T}$-independent blood-borne particulate antigens. Immunity. 14:617-629.

17. Storb, U., et al. 1986. Transgenic mice with mu and kappa genes encoding antiphosphorylcholine antibodies. J. Exp. Med. 164:627-641.

18. Erickson, L.D., Lin, L.L., Duan, B., Morel, L., and Noelle, R.J. 2003. A genetic lesion that arrests plasma cell homing to the bone marrow. Proc. Natl. Acad. Sci. U. S. A. 100:12905-12910.
19. Cancro, M.P. 2004. The BLyS family of ligands and receptors: an archetype for niche-specific homeostatic regulation. Immunol. Rev. 202:237-249.

20. Yu, G., et al. 2000. APRIL and TALL-I and receptors BCMA and TACI: system for regulating humoral immunity. Nat. Immunol. 1:252-256.

21. Lesley, R., et al. 2004. Reduced competitiveness of autoantigen-engaged $\mathrm{B}$ cells due to increased dependence on BAFF. Immunity. 20:441-453.

22. Thien, M., et al. 2004. Excess BAFF rescues selfreactive $B$ cells from peripheral deletion and allows them to enter forbidden follicular and marginal zone niches. Immunity. 20:785-798.

23. Gorelik, L., et al. 2004. Cutting edge: BAFF regulates CD21/35 and CD23 expression independent of its B cell survival function. J. Immunol. 172:762-766.

24. Ngo, V.N., Cornall, R.J., and Cyster, J.G. 2001. Splenic T zone development is B cell dependent. J. Exp. Med. 194:1649-1660.

25. Grammer, A.C., and Lipsky, P.E. 2003. B cell abnormalities in systemic lupus erythematosus. Arthritis Res. Ther. 5:S22-S27.

26. Shlomchik, M.J., Craft, J.E., and Mamula, M.J. 2001. From $\mathrm{T}$ to $\mathrm{B}$ and back again: positive feedback in systemic autoimmune disease. Nat. Rev. Immunol. 1:147-153.

27. Ng, L.G., et al. 2004. B cell-activating factor belonging to the TNF family (BAFF)- $\mathrm{R}$ is the principal BAFF receptor facilitating BAFF costimulation of circulating $\mathrm{T}$ and $\mathrm{B}$ cells. J. Immunol. 173:807-817.

28. Schiffer, L., et al. 2003. Short term administration of costimulatory blockade and cyclophosphamide induces remission of systemic lupus erythematosus nephritis in NZB/W F1 mice by a mechanism downstream of renal immune complex deposition. J. Immunol. 171:489-497.

29. Hoyer, B.F., et al. 2004. Short-lived plasmablasts and long-lived plasma cells contribute to chronic humoral autoimmunity in NZB/W mice. J. Exp. Med. 199:1577-1584.

30. Ingold, K., et al. 2005. Identification of proteoglycans as the APRIL-specific binding partners. J. Exp. Med. 201:1375-1383.

31. Groom, J., et al. 2002. Association of BAFF/BLyS overexpression and altered $\mathrm{B}$ cell differentiation with Sjogren's syndrome. J. Clin. Invest. 109:59-68. doi:10.1172/JCI200214121.

32. Gavin, A.L., et al. 2005. DeltaBAFF, a splice isoform of BAFF, opposes full-length BAFF activity in vivo in transgenic mouse models. J. Immunol. 175:319-328.

33. Gross, J.A., et al. 2001. TACI-Ig neutralizes molecules critical for B cell development and autoimmune disease: impaired B cell maturation in mice lacking BLyS. Immunity. 15:289-302.

34. Planelles, L., et al. 2004. APRIL promotes B-1 cellassociated neoplasm. Cancer Cell. 6:399-408.

35. Balazs, M., Martin, F., Zhou, T., and Kearney, J. 2002. Blood dendritic cells interact with splenic marginal zone B cells to initiate T-independent immune responses. Immunity. 17:341-352.
36. von Bulow, G.U., van Deursen, J.M., and Bram, R.J. 2001. Regulation of the T-independent humoral response by TACI. Immunity. 14:573-582.

37. Shulga-Morskaya, S., et al. 2004. B cell-activating factor belonging to the TNF family acts through separate receptors to support B cell survival and $\mathrm{T}$ cell-independent antibody formation. J. Immunol. 173:2331-2341

38. Varfolomeev, E., et al. 2004. APRIL-deficient mice have normal immune system development. Mol. Cell. Biol. 24:997-1006.

39. Tumang, J.R., Hastings, W.D., Bai, C., and Rothstein, T.L. 2004. Peritoneal and splenic B-1 cells are separable by phenotypic, functional, and transcriptomic characteristics. Eur. J. Immunol. 34:2158-2167.

40. Rahman, Z.S., Rao, S.P., Kalled, S.L., and Manser, T. 2003. Normal induction but attenuated progression of germinal center responses in BAFF and BAFF-R signaling-deficient mice. J. Exp. Med. 198:1157-1169.

41. Vora, K.A., et al. 2003. Cutting edge: germinal centers formed in the absence of B cell-activating factor belonging to the TNF family exhibit impaired maturation and function. J. Immunol. 171:547-551.

42. O'Connor, B.P., et al. 2004. BCMA is essential for the survival of long-lived bone marrow plasma cells. J. Exp. Med. 199:91-98.

43. Schneider, P., et al. 2001. Maturation of marginal zone and follicular B cells requires B cell activating factor of the tumor necrosis factor family and is independent of B cell maturation antigen. J. Exp. Med. 194:1691-1697.

44. Bernasconi, N.L., Traggiai, E., and Lanzavecchia, A. 2002. Maintenance of serological memory by polyclonal activation of human memory B cells. Science. 298:2199-2202.

45. Salzer, U., et al. 2005. Mutations in TNFRSF13B encoding TACI are associated with common variable immunodeficiency in humans. Nat. Genet. 37:820-828.

46. Mihara, M., et al. 2000. CTLA4Ig inhibits T celldependent B-cell maturation in murine systemic lupus erythematosus. J. Clin. Invest. 106:91-101.

47. Chan, O., Madaio, M.P., and Shlomchik, M.J. 1997. The roles of B cells in MRL/lpr murine lupus. Ann. N. Y. Acad. Sci. 815:75-87.

48. Steinbrink, K., Sorg, C., and Macher, E. 1996. Low zone tolerance to contact allergens in mice: a functional role for CD8+ T helper type 2 cells. J. Exp. Med. 183:759-768.

49. Wang, X., Huang, W., Mihara, M., Sinha, J., and Davidson, A. 2002. Mechanism of action of combined short-term CTLA4Ig and anti-CD40 ligand in murine systemic lupus erythematosus. J. Immunol. 168:2046-2053.

50. Sieckmann, D.G., Martin, E., Guelde, G., Longo, D.L., and Kenny, J.J. 1997. Anti-idiotype monoclonal antibodies specific for the MOPC167 antiphosphocholine transgene-encoded antibody. Hybridoma. 16:503-511. 